Received: 19 October 2018

Accepted: 19 February 2019

Published online: 11 March 2019
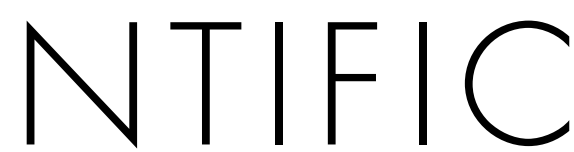

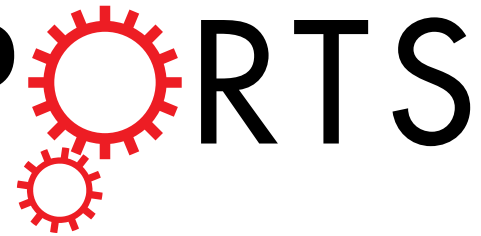

\title{
OPEN High-Electrical-Conductivity Multilayer Graphene Formed by Layer Exchange with Controlled Thickness and Interlayer
}

\author{
Hiromasa Murata ${ }^{1}$, Yoshiki Nakajima ${ }^{1}$, Noriyuki Saitoh ${ }^{2}$, Noriko Yoshizawa ${ }^{2}$, \\ Takashi Suemasu ${ }^{1} \&$ Kaoru Toko ${ }^{1,3}$
}

The layer exchange technique enables high-quality multilayer graphene (MLG) on arbitrary substrates, which is a key to combining advanced electronic devices with carbon materials. We synthesize uniform MLG layers of various thicknesses, $t$, ranging from $5 \mathrm{~nm}$ to $200 \mathrm{~nm}$ using $\mathrm{Ni}$-induced layer exchange at $800^{\circ} \mathrm{C}$. Raman and transmission electron microscopy studies show the crystal quality of MLG is relatively low for $t \leq 20 \mathrm{~nm}$ and dramatically improves for $t \geq 50 \mathrm{~nm}$ when we prepare a diffusion controlling $\mathrm{Al}_{2} \mathrm{O}_{3}$ interlayer between the $\mathrm{C}$ and Ni layers. Hall effect measurements reveal the carrier mobility for $t=50 \mathrm{~nm}$ is $550 \mathrm{~cm}^{2} / \mathrm{Vs}$, which is the highest Hall mobility in MLG directly formed on an insulator. The electrical conductivity $(2700 \mathrm{~S} / \mathrm{cm})$ also exceeds a highly oriented pyrolytic graphite synthesized at $3000^{\circ} \mathrm{C}$ or higher. Synthesis technology of MLG with a wide range of thicknesses will enable exploration of extensive device applications of carbon materials.

Multilayer graphene (MLG) has excellent characteristics, such as high electrical/thermal conductivities and current-carrying capacity exceeding that of $\mathrm{Cu}^{1-4}$. Therefore, application of MLG on arbitrary substrates is expected in various applications, including transparent electrodes, low-resistance wiring, and heat spreaders. As the required MLG thickness depends on the application, a technique for controlling the thickness of high-quality MLG film is essential.

High-quality graphene and MLG have been produced on arbitrary substrates using transfer techniques ${ }^{5}$ and chemical vapor deposition ${ }^{6-11}$. Some of these techniques can precisely control the numbers of graphene layers; however, there is difficulty forming thick MLG in the tens of nanometers. Against this backdrop, metal-induced solid-phase crystallization of amorphous carbon $(\mathrm{a}-\mathrm{C})$ or polymers has attracted increased attention owing to the direct synthesis of MLG on insulators ${ }^{12-26}$. Some of these techniques have allowed synthesis of thick $(>5 \mathrm{~nm})$ MLG by controlling the initial thickness of a- $\mathrm{C}^{17-26}$. However, a problem has existed with the uniformity of the MLG layer, which makes it difficult to systematically evaluate the electrical properties of such layers.

Metal-induced layer exchange (MILE) has been actively studied in the field of group-IV semiconductors, including $\mathrm{Si}^{27-31}, \mathrm{Ge}^{32-37}$, and $\mathrm{SiGe}^{38,39}$. In MILE, an amorphous semiconductor layer crystallizes through "layer exchange" between the amorphous layer and a catalyst metal layer. The thickness design of the initial metal layer can easily control the resulting semiconductor layer ${ }^{27,31,35}$. We found the layer exchange occurs in the Co-C and $\mathrm{Ni}-\mathrm{C}$ systems and fabricated a uniform $50-\mathrm{nm}$-thick MLG on an insulator ${ }^{40,41}$. Furthermore, we employed a diffusion controlling interlayer (IL) between $\mathrm{C}$ and $\mathrm{Ni}$, which suppresses the nucleation of the MLG and results in enlargement of MLG grains ${ }^{42}$. In MILE, the initial metal thickness strongly influences the crystallinity of the resulting semiconductor layer ${ }^{31,38}$. This study first clarifies the effect of film thickness on the crystallinity and electrical properties of MLG on an insulator. The result shows a carrier mobility of $550 \mathrm{~cm}^{2} / \mathrm{Vs}$ and an electrical conductivity of $2700 \mathrm{~S} / \mathrm{cm}$; the highest values among most MLG layers directly formed on insulators.

${ }^{1}$ Institute of Applied Physics, University of Tsukuba, 1-1-1 Tennodai, Tsukuba, Ibaraki, 305-8573, Japan. ${ }^{2}$ Electron Microscope Facility, TIA, AIST, 16-1 Onogawa, Tsukuba, 305-8569, Japan. ${ }^{3}$ PRESTO, Japan Science and Technology Agency, 4-1-8 Honcho, Kawaguchi, Saitama, 332-0012, Japan. Correspondence and requests for materials should be addressed to K.T. (email: toko@bk.tsukuba.ac.jp) 

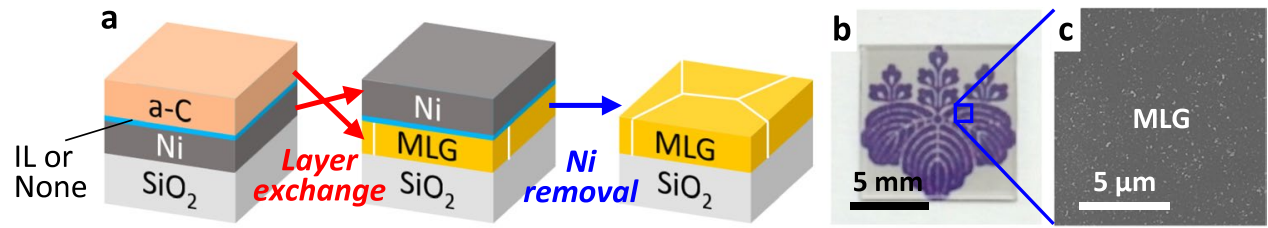

Figure 1. Typical example of layer exchange. (a) Schematic of the sample preparation procedure. (b) Photograph and (c) SEM image of the sample for $t=5 \mathrm{~nm}$ without IL after Ni removal.
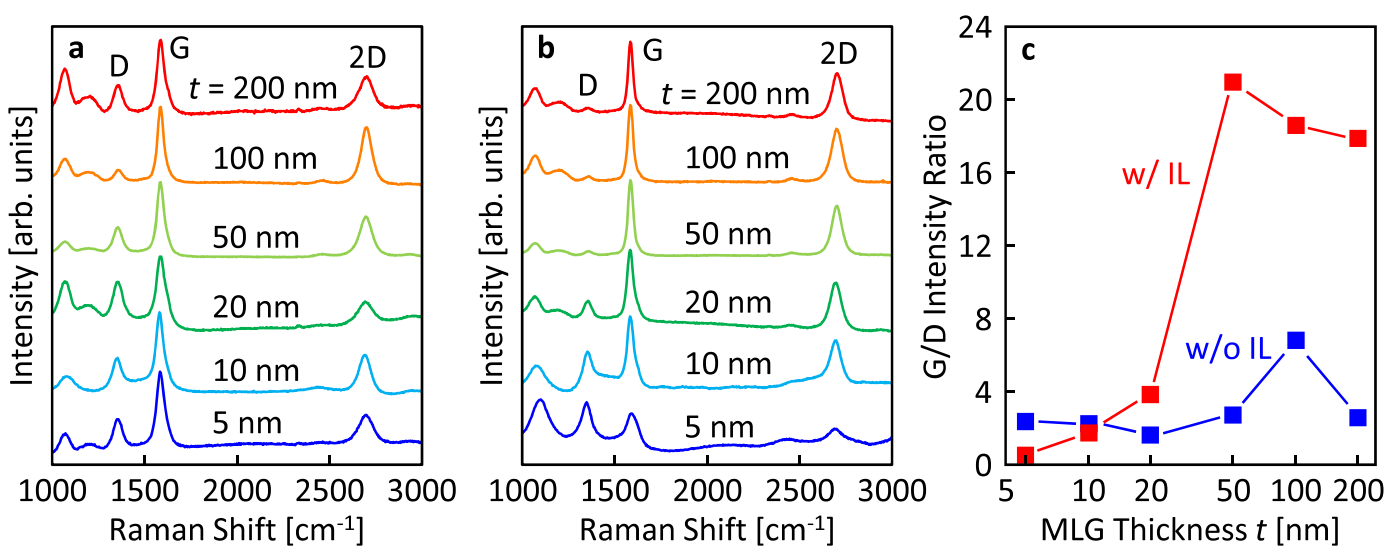

Figure 2. Raman study of MLG formed by layer exchange. Raman spectra obtained from back side of the samples before Ni removal (a) without and (b) with the IL. (c) G/D intensity ratio of the samples determined by the Raman spectra shown in $(\mathbf{a}, \mathbf{b})$, as a function of $t$.

\section{Results}

As shown in Fig. 1a, all samples started with a stacked structure of an a-C/Ni/SiO 2 substrate and were then annealed at $800^{\circ} \mathrm{C}$ for $1 \mathrm{~h}$ in ambient Ar. We prepared the samples with and without a diffusion controlling $\mathrm{Al}_{2} \mathrm{O}_{3}$ IL, which improves the crystal quality of $\mathrm{MLG}^{42}$. The thickness of the initial Ni layer determines the resulting MLG thickness, $t$, after layer exchange ${ }^{41,42}$. We varied $t$ from $5 \mathrm{~nm}$ to $200 \mathrm{~nm}$ to investigate the effects of $t$ on the crystal and electrical properties of the MLG. For all samples, MLG layers were obtained on the substrate via layer exchange. As representatively shown in Fig. 1b, thin MLG layers $(t \leq 10 \mathrm{~nm})$ exhibited transparency. The scanning electron microscopy (SEM) image in Fig. 1c shows that although the MLG layer has submicron-size voids, it covers nearly the entire substrate. We note the Ni concentration in the MLG layer is below the detection limit of $\operatorname{EDX}(\sim 1 \%)$.

Figure 2a,b show that Raman spectra of the back side of the samples have peaks at approximately 1350, 1580, and $2700 \mathrm{~cm}^{-1}$, corresponding to the D (disordered mode), G (graphitic mode), and 2D (D mode overtone) peaks in the graphitic structure, respectively. This means layer exchange between the $\mathrm{C}$ and Ni layers occurred and MLG formed on the $\mathrm{SiO}_{2}$ glass substrate in all samples. In the Raman spectra, the intensity ratio of the $\mathrm{G}$ to D peaks $\left(I_{\mathrm{G}} / I_{\mathrm{D}}\right)$ in the spectra corresponds to the crystal quality of MLG ${ }^{43}$. Figure $2 \mathrm{c}$ indicates the $I_{\mathrm{G}} / I_{\mathrm{D}}$ ratio strongly depends on $t$, and with or without the IL. For the samples without the IL, the $I_{\mathrm{G}} / I_{\mathrm{D}}$ ratio shows the highest value of 7 at $t=100 \mathrm{~nm}$. For the samples with the IL, the $I_{\mathrm{G}} / I_{\mathrm{D}}$ ratio is as low as for the samples without the IL for $t \leq 20 \mathrm{~nm}$, while it dramatically increases for $t \geq 50 \mathrm{~nm}$ and exceeds 20 . The crystal quality of the MLG, estimated from the $I_{\mathrm{G}} / I_{\mathrm{D}}$ ratio, likely reflects the contamination from the substrate and the grain size enlargement by inserting the $\mathrm{IL}^{42}$. Although the $I_{\mathrm{G}} / I_{\mathrm{D}}$ ratio of 20 is still lower than that of MLG formed by the transfer technique or high-temperature $\mathrm{CVD}^{4}$, this value is the highest among MLG synthesized on insulators by metal-induced solid-phase crystallization ${ }^{18,19,23,25}$.

The detailed cross-sectional structure of the sample for $t=10 \mathrm{~nm}$, which is a typical thin transparent film, was investigated using an analytical transmission electron microscope (TEM). Figure 3a shows the MLG formed on the entire substrate. Figure 3b,c show Ni on the MLG layer is particle-shaped, whereas the sample with $t=50 \mathrm{~nm}$ has a continuous Ni layer ${ }^{41,42}$. Considering that the MLG layer is uniformly formed, the Ni particles are likely attributed to the agglomeration of a thin Ni layer moved on MLG after layer exchange. Figure 3d,e show $\{002\}$ oriented MLG forms on the $\mathrm{SiO}_{2}$ substrate in both the Ni-contacted and non-Ni-contacted regions. The MLG in the non-Ni-contacted region is slightly thinner than that in the Ni-contacted region, probably because of the $\mathrm{C}$ evaporation during annealing. Figure 3d,e indicate the crystal structure of the thin MLG is disordered compared with the sample with an $\mathrm{IL}^{42}$. We note that, for $t=50 \mathrm{~nm}$, the grain size of the MLG layer without an IL was a few hundred $\mathrm{nm}$ and that with an IL was a few $\mu \mathrm{m}^{41,42}$. These results account well for the Raman study (Fig. 2c). For high-quality MLG, such as that synthesized by thermal decomposition of a SiC substrate, TEM observation can identify the stacking structure (e.g. AA and AB stacking) ${ }^{44,45}$. Unfortunately, because the current MLG is 


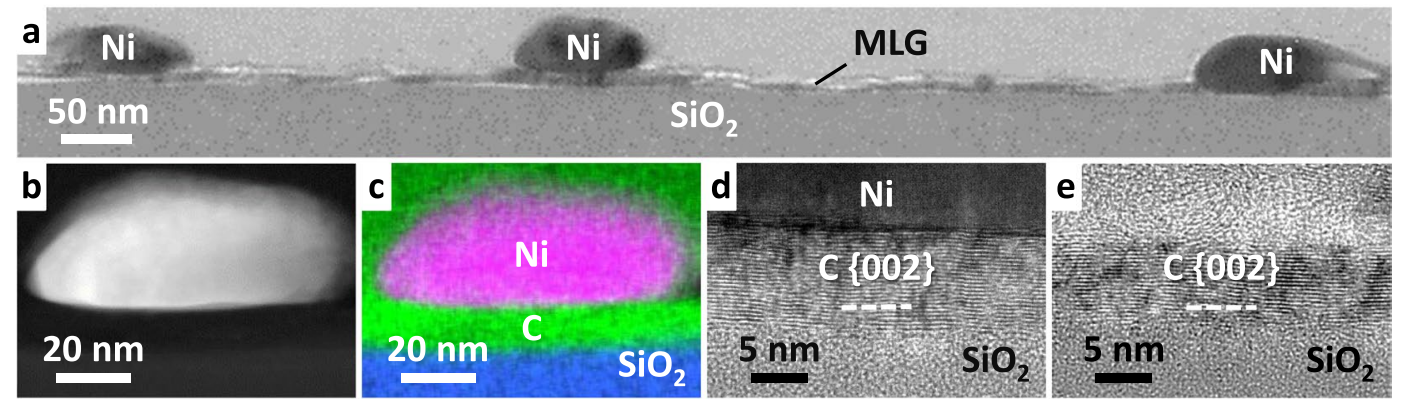

Figure 3. Characterization of the cross-section of the sample for $t=10 \mathrm{~nm}$ without the IL before Ni removal. (a) Bright-field TEM image. (b) HAADF-STEM image and (c) EDX elemental map of an Ni-contacted region. High-resolution lattice images of the MLG layer showing (d) an Ni-contacted region and (e) a non-Nicontacted region.
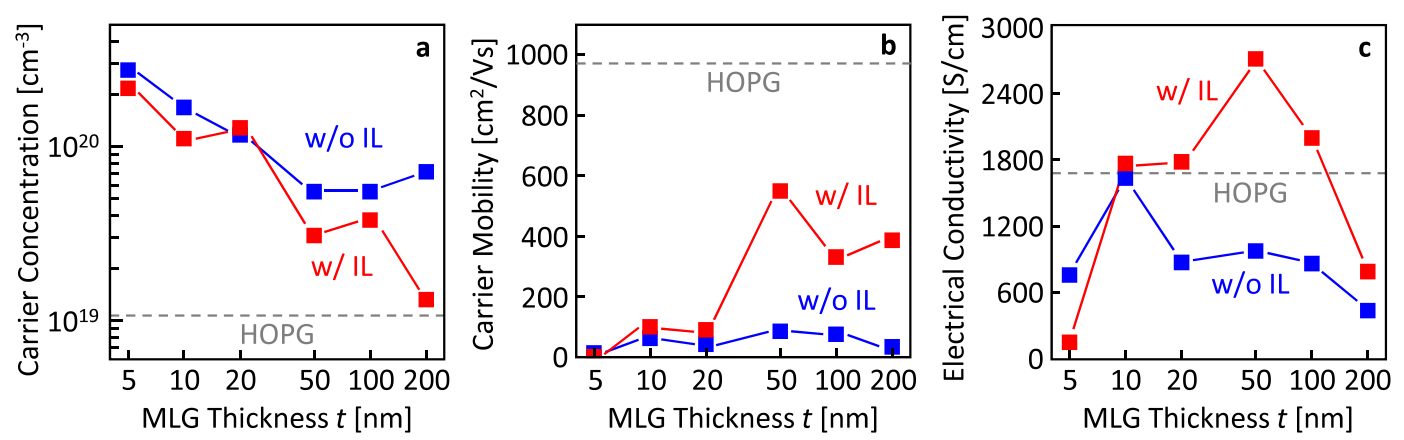

Figure 4. Electrical properties of the MLG layers after Ni removal as a function of $t$. (a) Carrier concentration. (b) Carrier mobility. (c) Electrical conductivity. The data of a HOPG are shown as dotted lines.

small-grained polycrystalline where the grains are randomly oriented in the in-plane direction ${ }^{42}$, it is difficult to identify the stacking.

Figure 4 shows the electrical properties of the MLG strongly depend on $t$, and with or without the IL. Figure $4 \mathrm{a}$ shows the carrier concentration of the MLG decreases with the increase of $t$ and approaches the value of a highly oriented pyrolytic graphite (HOPG) with a low mosaic degree of $0.4^{\circ}$, especially for the sample with the IL. Figure $4 \mathrm{~b}$ shows the carrier mobility dramatically increases for the $t \geq 50 \mathrm{~nm}$ samples with the IL, whereas it remains low in the whole range of $t$ for the samples without the IL. The carrier mobility exhibits the maximum value of $550 \mathrm{~cm}^{2} / \mathrm{Vs}$ for the $t=50 \mathrm{~nm}$ sample with the IL, which is the highest Hall mobility among MLGs directly formed on an insulator. Figure $4 \mathrm{c}$ shows the behavior of the electrical conductivity reflects that of the carrier concentration and carrier mobility. The electrical conductivity exhibits the maximum value of $2700 \mathrm{~S} / \mathrm{cm}$ for the $t=50 \mathrm{~nm}$ sample with the IL, which exceeds that of the HOPG synthesized at $3000^{\circ} \mathrm{C}$ or higher.

\section{Discussion}

We have controlled the thickness of MLG on an insulator formed by layer exchange with and without the IL, resulting in a high electrical conductivity. Considering the fact that the behavior of the carrier mobility (Fig. 4b) is the same as that of the $I_{\mathrm{G}} / I_{\mathrm{D}}$ ratio in the Raman spectra (Fig. 2c), the carrier mobility clearly reflects the crystal quality of the MLG. The carrier mobility, that is, the crystal quality of the MLG is much higher than any other MLG directly formed on an insulator; however, does not reach the HOPG. The electrical conductivity of the MLG exceeds that of HOPG with the aid of autodoping. Although the reasons for the excessive carriers for the MLG are still unclear, when we assume defects in MLG produce carriers, the carrier concentration behavior is almost consistent with the Raman study.

In conclusion, the crystal quality and electrical properties of the MLG, formed on an insulator by layer exchange at $800^{\circ} \mathrm{C}$, strongly depended on $t$, especially when preparing the IL. The crystal quality was relatively low for $t \leq 20 \mathrm{~nm}$; however, it dramatically improved for $t \geq 50 \mathrm{~nm}$ with the IL. As a result, the sample for $t=50 \mathrm{~nm}$ with the IL exhibited the maximum carrier mobility $\left(550 \mathrm{~cm}^{2} / \mathrm{Vs}\right)$, and the highest Hall mobility among MLG directly formed on an insulator. The electrical conductivity $(2700 \mathrm{~S} / \mathrm{cm})$ even exceeded the high-quality HOPG (mosaic degree: $0.4^{\circ}$ ). Thus, the layer exchange method allows us to form MLG with a wide range of thicknesses on arbitrary substrates. This opens the door for a broad range of applications that combine advanced electronic devices with carbon materials. 


\section{Methods}

Sample preparation. Ni thin films with thicknesses of 5, 10, 20, 50, 100, and $200 \mathrm{~nm}$ were prepared on $\mathrm{SiO}_{2}$ glass substrates. Subsequently, $\mathrm{Al}_{2} \mathrm{O}_{3}$ (thickness: $2 \mathrm{~nm}$ ) was employed as the diffusion control $\mathrm{IL}^{42}$. Then, a-C thin films were prepared, wherein the thickness ratio of $\mathrm{C}: \mathrm{Ni}=3: 2$ (e.g., $\mathrm{C}: \mathrm{Ni}=300: 200 \mathrm{~nm}$ ). All depositions were carried out using radio-frequency (RF) magnetron sputtering (base pressure: $3.0 \times 10^{-4} \mathrm{~Pa}$ ) with an Ar plasma. During the deposition, the substrate temperature was room temperature for $\mathrm{Al}_{2} \mathrm{O}_{3}$ and $200^{\circ} \mathrm{C}$ for $\mathrm{Ni}$ and $\mathrm{C}$. The $\mathrm{RF}$ power was set to $100 \mathrm{~W}$ for $\mathrm{C}$ and $50 \mathrm{~W}$ for $\mathrm{Ni}$ and $\mathrm{Al}_{2} \mathrm{O}_{3}$. For comparison, we prepared the samples without the $\mathrm{Al}_{2} \mathrm{O}_{3} \mathrm{IL}$. Samples were annealed at $800^{\circ} \mathrm{C}$ for $1 \mathrm{~h}$ in an $\mathrm{Ar}$ ambient to induce layer exchange growth. The sample was dipped in a diluted $\mathrm{HNO}_{3}$ solution $\left(10 \% \mathrm{HNO}_{3}\right)$ for 30 min to remove Ni moved to the top layer.

Material characterization. SEM analyses were performed using a JEOL JSM-7001F with an EDX spectrometer (JEOL JEO-2300). Raman spectroscopy was performed using a JASCO NRS-5100, wherein the laser wavelength was $532 \mathrm{~nm}$ and the spot size was $5 \mu \mathrm{m}$. TEM analyses were performed using an analytical TEM, FEI Tecnai Osiris, operating at $200 \mathrm{kV}$, equipped with an EDX spectrometer (FEI Super-X system). The cross-sectional TEM sample was prepared using the conventional focused ion beam method. Hall effect measurement was performed with the Van der Pauw method using the Bio-Rad HL5500PC system. Carrier mobility, carrier concentration, and electrical conductivity were averaged over five measurements for each sample.

\section{Data Availability}

The data used in this study are available upon reasonable request from the corresponding author K.T. (toko@ bk.tsukuba.ac.jp).

\section{References}

1. Biswas, S. \& Drzal, L. T. Multilayered nano-architecture of variable sized graphene nanosheets for enhanced supercapacitor electrode performance. ACS Appl. Mater. Interfaces 2, 2293-2300 (2010).

2. Balandin, A. A. Thermal properties of graphene and nanostructured carbon materials. Nat. Mater. 10, 569-581 (2011).

3. Murali, R., Yang, Y., Brenner, K., Beck, T. \& Meindl, J. D. Breakdown current density of graphene nanoribbons. Appl. Phys. Lett. 94, 243114 (2009).

4. Kim, K. S. et al. Large-scale pattern growth of graphene films for stretchable transparent electrodes. Nature 457, 706-710 (2009).

5. Novoselov, K. S. et al. Electric field effect in atomically thin carbon films. Science 306, 666-669 (2004).

6. Su, C.-Y. et al. Direct formation of wafer scale graphene thin layers on insulating substrates by chemical vapor deposition. Nano Lett. 11, 3612-3616 (2011)

7. Kato, T. \& Hatakeyama, R. Direct growth of doping-density-controlled hexagonal graphene on $\mathrm{SiO}_{2}$ substrate by rapid-heating plasma CVD. ACS Nano 6, 8508-8515 (2012).

8. Yen, W.-C. C. et al. Direct growth of self-crystallized graphene and graphite nanoballs with Ni vapor-assisted growth: from controllable growth to material characterization. Sci. Rep. 4, 4739 (2014).

9. Murakami, K. et al. Direct synthesis of large area graphene on insulating substrate by gallium vapor-assisted chemical vapor deposition. Appl. Phys. Lett. 106, 093112 (2015).

10. Chugh, S. et al. Comparison of graphene growth on arbitrary non-catalytic substrates using low-temperature PECVD. Carbon 93, 393-399 (2015)

11. Ueno, K., Ichikawa, H. \& Uchida, T. Effect of current stress during thermal CVD of multilayer graphene on cobalt catalytic layer. Jpn. J. Appl. Phys. 55, 04EC13 (2016)

12. Peng, Z., Yan, Z., Sun, Z. \& Tour, J. M. Direct growth of bilayer graphene on $\mathrm{SiO}_{2}$ substrates by carbon diffusion through nickel. ACS Nano 5, 8241-8247 (2011).

13. Kwak, J. et al. Near room-temperature synthesis of transfer-free graphene films. Nat. Commun. 3, 645 (2012).

14. Banno, K. et al. Transfer-free graphene synthesis on insulating substrates via agglomeration phenomena of catalytic nickel films. Appl. Phys. Lett. 103, 082112 (2013).

15. Xiong, W. et al. Solid-state graphene formation via a nickel carbide intermediate phase. RSC Adv. 5, 99037-99043 (2015).

16. Berman, D. et al. Metal-induced rapid transformation of diamond into single and multilayer graphene on wafer scale. Nat. Commun. 7, 12099 (2016)

17. Vishwakarma, R. et al. Transfer free graphene growth on $\mathrm{SiO}_{2}$ substrate at $250^{\circ} \mathrm{C}$. Sci. Rep. 7, 43756 (2017).

18. Byun, S. J. et al. Graphenes converted from polymers. J. Phys. Chem. Lett. 2, 493-497 (2011).

19. Gumi, K., Ohno, Y., Maehashi, K., Inoue, K. \& Matsumoto, K. Direct Synthesis of Graphene on $\mathrm{SiO}_{2}$ Substrates by Transfer-Free Processes. Jpn. J. Appl. Phys. 51, 06FD12 (2012).

20. Weatherup, R. S. et al. Introducing carbon diffusion barriers for uniform, high-quality graphene growth from solid sources. Nano Lett. 13, 4624-4631 (2013).

21. Tamaoki, M., Imaeda, H., Kishimoto, S. \& Mizutani, T. Transfer-free fabrication of graphene field effect transistor arrays using solidphase growth of graphene on $\mathrm{SiO}_{2} / \mathrm{Si}$ substrate. Appl. Phys. Lett. 103, 183114 (2013).

22. Tanaka, H., Obata, S. \& Saiki, K. Reduction of graphene oxide at the interface between a Ni layer and a SiO $\mathrm{S}_{2}$ substrate. Carbon $\mathbf{5 9}$, 472-478 (2013).

23. Sato, M. et al. Intercalated multilayer graphene wires and metal/multilayer graphene hybrid wires obtained by annealing sputtered amorphous carbon. Jpn. J. Appl. Phys. 53, 04EB05 (2014).

24. Kosaka, M., Takano, S., Hasegawa, K. \& Noda, S. Direct synthesis of few- and multi-layer graphene films on dielectric substrates by "etching-precipitation" method. Carbon 82, 254-263 (2015).

25. Zhuo, Q. Q. et al. Transfer-free synthesis of doped and patterned graphene films. ACS Nano 9, 594-601 (2015).

26. Yamada, J., Ueda, Y., Maruyama, T. \& Naritsuka, S. Direct growth of multilayer graphene by precipitation using W capping layer. Jpn. J. Appl. Phys. 55, $100302(2016)$.

27. Nast, O., Puzzer, T., Koschier, L. M., Sproul, A. B. \& Wenham, S. R. Aluminum-induced crystallization of amorphous silicon on glass substrates above and below the eutectic temperature. Appl. Phys. Lett. 73, 3214-3216 (1998).

28. Sarikov, A. et al. A kinetic simulation study of the mechanisms of aluminum induced layer exchange process. J. Appl. Phys. 107, $114318(2010)$.

29. Wang, Z., Gu, L., Jeurgens, L. P. H., Phillipp, F. \& Mittemeijer, E. J. Real-time visualization of convective transportation of solid materials at nanoscale. Nano Lett. 12, 6126-6132 (2012).

30. Birajdar, B. I., Antesberger, T., Butz, B., Stutzmann, M. \& Spiecker, E. Direct in situ transmission electron microscopy observation of Al push up during early stages of the Al-induced layer exchange. Scr. Mater. 66, 550-553 (2012).

31. Toko, K. et al. Selective formation of large-grained, (100)- or (111)-oriented Si on glass by Al-induced layer exchange. J. Appl. Phys. 115, 094301 (2014). 
32. Toko, K. et al. Highly (111)-oriented Ge thin films on insulators formed by Al-induced crystallization. Appl. Phys. Lett. 101, 072106 (2012).

33. Park, J.-H., Kasahara, K., Hamaya, K., Miyao, M. \& Sadoh, T. High carrier mobility in orientation-controlled large-grain $(\geq 50 \mu \mathrm{m})$ Ge directly formed on flexible plastic by nucleation-controlled gold-induced-crystallization. Appl. Phys. Lett. 104, 252110 (2014).

34. Toko, K. et al. Low-temperature $\left(180^{\circ} \mathrm{C}\right)$ formation of large-grained $\mathrm{Ge}(111)$ thin film on insulator using accelerated metal-induced crystallization. Appl. Phys. Lett. 104, 022106 (2014).

35. Toko, K., Nakazawa, K., Saitoh, N., Yoshizawa, N. \& Suemasu, T. Improved surface quality of the metal-induced crystallized Ge seed layer and its influence on subsequent epitaxy. Cryst. Growth Des. 15, 1535-1539 (2015).

36. Higashi, H. et al. Electrical properties of pseudo-single-crystalline Ge films grown by Au-induced layer exchange crystallization at $250^{\circ}$ C. J. Appl. Phys. 123, 215704 (2018).

37. Yoshimine, R., Toko, K., Saitoh, N., Yoshizawa, N. \& Suemasu, T. Silver-induced layer exchange for polycrystalline germanium on a flexible plastic substrate. J. Appl. Phys. 122, 215305 (2017).

38. Kurosawa, M., Kawabata, N., Sadoh, T. \& Miyao, M. Enhanced interfacial-nucleation in Al-induced crystallization for (111) oriented $\mathrm{Si}_{1-x} \mathrm{Ge}_{x}(0 \leq x \leq 1)$ films on insulating substrates. ECS J. Solid State Sci. Technol. 1, 144-147 (2012).

39. Toko, K., Kusano, K., Nakata, M. \& Suemasu, T. Low temperature synthesis of highly oriented p-type $\mathrm{Si}_{1-x} \mathrm{Ge}_{x}(x: 0-1)$ on an insulator by Al-induced layer exchange. J. Appl. Phys. 122, 155305 (2017).

40. Murata, H., Toko, K. \& Suemasu, T. Multilayer graphene on insulator formed by Co-induced layer exchange. Jpn. J. Appl. Phys. 56, 05DE03 (2017).

41. Murata, H., Toko, K., Saitoh, N., Yoshizawa, N. \& Suemasu, T. Direct synthesis of multilayer graphene on an insulator by Ni-induced layer exchange growth of amorphous carbon. Appl. Phys. Lett. 110, 033108 (2017).

42. Murata, H., Saitoh, N., Yoshizawa, N., Suemasu, T. \& Toko, K. High-quality multilayer graphene on an insulator formed by diffusion controlled Ni-induced layer exchange. Appl. Phys. Lett. 111, 243104 (2017).

43. Chu, P. K. \& Li, L. Characterization of amorphous and nanocrystalline carbon films. Mater. Chem. Phys. 96, 253-277 (2006).

44. Borysiuk, J. et al. Role of structure of C-terminated $4 \mathrm{H}-\mathrm{SiC}(000-1)$ surface in growth of graphene layers: Transmission electron microscopy and density functional theory studies. Phys. Rev. B. 85, 045426 (2012).

45. Borysiuk, J. et al. Structural defects in epitaxial graphene layers synthesized on C-terminated 4H-SiC (000-1) surface-Transmission electron microscopy and density functional theory studies. J. Appl. Phys. 115, 054310 (2014).

\section{Acknowledgements}

This work was financially supported by JSPS KAKENHI (No. 18K18844), JSPS Research Fellow (No. 18J20904), Advanced Technology Institute Research Grants (RG2906), and JST PRESTO (No. JPMJPR17R7). The authors are grateful to Dr. T. Sakurai at the University of Tsukuba for assistance with the Hall effect measurements. Some experiments were conducted at the International Center for Young Scientists at NIMS and the Nanotechnology Platform at the University of Tsukuba and AIST.

\section{Author Contributions}

K.T. conceived and designed the experiments. H.M. and Y.N. conducted the sample fabrication and the majority of the analyses. N.S. and N.Y conducted the TEM analyses. K.T. and T.S. managed and supervised the research project. All authors discussed the results and commented on the manuscript.

\section{Additional Information}

Competing Interests: The authors declare no competing interests.

Publisher's note: Springer Nature remains neutral with regard to jurisdictional claims in published maps and institutional affiliations.

(c) (i) Open Access This article is licensed under a Creative Commons Attribution 4.0 International License, which permits use, sharing, adaptation, distribution and reproduction in any medium or format, as long as you give appropriate credit to the original author(s) and the source, provide a link to the Creative Commons license, and indicate if changes were made. The images or other third party material in this article are included in the article's Creative Commons license, unless indicated otherwise in a credit line to the material. If material is not included in the article's Creative Commons license and your intended use is not permitted by statutory regulation or exceeds the permitted use, you will need to obtain permission directly from the copyright holder. To view a copy of this license, visit http://creativecommons.org/licenses/by/4.0/.

(C) The Author(s) 2019 